\title{
IS THERE A ROLE FOR CONSERVATIVE TREATMENT IN THOSE WITH UNILATERAL TUBAL OCCLUSION?
}

Steven Robert Lindheim

Financial Disclosure: The authors do not have any potential conflicts of interest.

\begin{abstract}
Objective-HSG is the accepted standard to diagnose tubal patency. In contrast to bilateral tubal occlusion where therapy is directed towards laparoscopic correction or IVF, treatment of unilateral tubal occlusion (UTO) is less clear, including conservative OI and IUI directed towards the patent tube. We assessed the value of conservative OIIUI and pregnancy outcomes in those with UTO. Methods: We evaluated patients diagnosed on HSG with UTO $(n=24)$ (proximal $[n=7]$ and mid-distal or distal occlusion $[n=17])$. Inclusion included women <38 years; regular menstrual cycles; normal sperm parameters; and normal spill from 1 fallopian tube on HSG. Controls underwent donor insemination ( $\mathrm{n}=87$ in 275 cycles) with bilateral tubal spill. Treatment included LH testing and time intercourse $(n=5)$ or OI-IUI with Clomiphene Citrate or Letrazole $(n=19$ in 36 cycles). All treatment cycles were monitored by ultrasound; hCG was given when lead follicle size reached $>18 \mathrm{~mm}$ (unless recruited follicle on obstructed side); and IUI was performed 24-36 hours later. The primary outcome measured was clinical pregnancy (CP). Results: Baseline demographics including age $(32.2 \pm 4[ \pm \mathrm{SD}]$ vs $33.4 \pm 2$ yrs, p-NS) and BMI (27.9 \pm 7 vs $28.2 \pm 8 \mathrm{~kg} / \mathrm{m}^{2}$, p-NS) were similar between UTO and control groups. Between HSG and treatment, spontaneous pregnancy occurred in $5(21 \%)$ women with UTO (1 proximal, 4 distal). In those undergoing OI-IUI treatment, CP rates/patient (32\%, $\mathrm{n}=6 / 19$ and $24 \%, \mathrm{n}=21 / 87, \mathrm{p}=0.56)$ and $\mathrm{CP} /$ cycle $(17 \%, 6 / 36$ and $8 \%, 21 / 275, \mathrm{p}=0.10)$ were similar for UTO and control groups. Overall, CP occurred in $2(29 \%)$ and $9(53 \%)$ patients with proximal and mid-distal or distal UTO, $\mathrm{p}=0.005$, respectively. Twenty-nine ( $81 \%)$ cycles recruited a dominant follicle on the patent side $(19 \% \mathrm{CP} /$ cycle $)$, in contrast no pregnancies occurred $(0 \%, 0 / 7)$ if recruitment occurred on the side of UTO, $\mathrm{p}=0.3$. Conclusions: Pregnancy rates are not compromised in women with UTO and conservative treatment with OI-IUI appears justified as a first line approach, obviating more aggressive therapies including laparoscopy and IVF.
\end{abstract}

Keywords - infertility; unilateral tubal occlusion; ovulation induction; laparoscopy 


\section{Section 1: Introduction}

Hysterosalpingogram (HSG) remains the current standard for assessing tubal patency in patients with unexplained infertility. HSG serves to screen for abnormalities in uterine anatomy and for tubal occlusion. In the case of bilateral tubal occlusion, treatment involves assisted reproductive methods such as in vitro fertilization (IVF). For patients with unilateral tubal occlusion (UTO), however, three treatment options have been reviewed including laparoscopic surgical correction; IVF; and ovulation induction (OI) with follicle recruitment on the patent side and intrauterine insemination (IUI).

OI-IUI, the most conservative approach, may involve the use of oral therapy with Clomiphene Citrate (CC) or injected gonadotropins (Gn). Gn are very effective at follicle recruitment, but have been known to cause ovarian hyperstimulation syndrome (OHSS), multiple gestation, and ectopic pregnancy (American Society for Reproductive Medicine 2012). The benefits of oral treatment are lower economic cost and fewer potential health risks, though oral treatments are less successful at recruiting follicles from the side of the patent fallopian tube.

In addition to the array of treatment options, the situation of UTO is further complicated by the fact that HSG has a demonstrated false positive rate as high as 25\% (Saunders 2011). False positive results are most often caused by spasms and air bubbles, and can potentially indicate more aggressive treatment than necessary, incurring unnecessary economic costs and medical risks.

While previous research supports the use of either $\mathrm{Gn}$ or CC in OI-IUI for patients with UTO, there is debate about the efficacy of OI-IUI in patients with distal UTO (Farhi 2011, Lin 2013, Yi 2012, Berker 2014, Saitou 2010, Ebrahimi 2011). This present study sought to determine the effectiveness of OI-IUI using oral therapy rather than $\mathrm{Gn}$, in cases of proximal UTO and distal UTO. We hypothesize that there is value to using oral therapy for OI-IUI in patients with either proximal or distal UTO.

\section{Section 2: Materials and Methods}

This study was a retrospective case controlled review conducted with approval by the Institutional Review Board and cooperation with standards set by the Health Information Portability and Accountability Act (HIPAA). The manuscript was designed according to the STROBE guidelines.

\section{Section 2.1 Design}

From January 2007 to December 2014, a retrospective chart review was undertaken of patients diagnosed on HSG with UTO $(n=24)$, including both proximal $(\mathrm{n}=7)$ and mid-distal or distal occlusion $(n=17)$.

\section{Section 2.2 Subjects and Protocol}

Inclusion criteria for the study group $(\mathrm{n}=24)$ included: (1) females less than 38 years of age; (2) regular menstrual cycles; (3) partners with normal sperm parameters; and (4) findings on HSG of 
a normal uterine cavity and normal spill from one fallopian tube. Those with hydrosalpinx from the occluded side were excluded due to the possible toxicity to embryo development (Strandell 2002). The control group consisted of patients $(n=87)$ undergoing donor insemination for unexplained infertility ( $n=275$ cycles). The control group met the same inclusion criteria as the study group, except control participants demonstrated bilateral tubal spill on HSG.

Following diagnosis, the treatment course involved LH Testing combined with Timed Intercourse (TIC) $(n=5)$, or oral $\mathrm{CC}$ or Letrazole followed by IUI ( $\mathrm{n}=19$ patients, 36 cycles). Ovulation induction was performed using CC $100 \mathrm{mg}$ or Letrazole $5 \mathrm{mg}$ to $7.5 \mathrm{mg}$ starting on cycle days 2 to 5 for five days. Repeat ultrasound was performed 3-4 days upon completion of ovarian stimulation. When the lead follicle size was larger than $18 \mathrm{~mm}$, hCG was given. IUI was performed 24-36 hours later, unless the follicle was recruited on the obstructed side and patients were instructed to have timed intercourse. Pregnancyßwas confirmed by serial serum -hCG 14 to 16 days following the IUI or with a skipped menses. Clinical and ongoing pregnancies were defined as the presence of an intrauterine gestational sac at 6 weeks and 12 weeks of gestation, respectively.

Section 2.3 Measured Outcomes and Statistical Analysis

Primary outcomes measured were demographics and clinical pregnancy (CP) per patient and per number of treated cycles. Secondary outcomes measured were differences between proximal and distal occlusion outcomes. Statistical analysis was performed using the SPSS statistical package, version 16.0 (SPSS Inc., Chicago, IL). Data were expressed as mean \pm SD. Unpaired t-test was used to test the significance of differences in means between groups and chisquare test was used to assess the significance of categorical comparisons. Odds ratio (OR) and corresponding confidence intervals $(95 \%$ CI $)$ were estimated. Significance was defined as a p-value $<0.05$.

\section{Section 3: Results}

Baseline demographics including age, BMI, and Total Motile Counts (TMC) were similar between UTO and control groups. The demographic characteristics of study participants are summarized in Figure 1. 


\begin{tabular}{|c|c|c|c|}
\hline \multicolumn{4}{|c|}{ Figure 1. Demographic Characteristics of Study Participants } \\
\hline & $\begin{array}{c}\text { UTO study group } \\
(\mathbf{n = 2 4})\end{array}$ & $\begin{array}{c}\text { Control group } \\
(\mathbf{n = 8 7})\end{array}$ & p-value \\
\hline Age (years) & $32.2 \pm 4$ & $33.4 \pm 2$ & NS \\
\hline BMI $\left(\mathrm{kg} / \mathrm{m}^{2}\right)$ & $27.9 \pm 7$ & $28.2 \pm 8$ & NS \\
\hline Gravidity & $1.2 \pm 0.4$ & $1.4 \pm 0.2$ & NS \\
\hline Parity & $0.5 \pm 0.1$ & $0.6 \pm 0.1$ & NS \\
\hline TMC $\left(\mathrm{x} 10^{6}\right)$ & $29.5 \pm 18.4$ & $30.7 \pm 16.5$ & NS \\
\hline Proximal Occlusion & $7(29 \%)$ & --- & --- \\
\hline $\begin{array}{c}\text { Mid-Distal/Distal } \\
\text { Occlusion }\end{array}$ & $17(71 \%)$ & - & -- \\
\hline
\end{tabular}

Between HSG and treatment, natural pregnancy occurred in $5(21 \%)$ women with UTO (1 with proximal occlusion and 4 with mid-distal or distal occlusion), and 0 (0\%) patients in the control group. Overall, clinical pregnancies were greater in those with UTO (46\%, 11/24 patients) (timed intercourse and OI-IUI) than in those undergoing donor insemination (24\%, $\mathrm{n}=21 / 87) \quad(\mathrm{p}=0.045 ; \mathrm{OR}=2.65 ; 95 \%$ CI: 1.04-6.8). In those undergoing treatment, CPR/patient $(32 \%, \mathrm{n}=6 / 19$ and $24 \%, \mathrm{n}=21 / 87, \mathrm{p}=0.56 ; \mathrm{OR}=1.45$; 95\% CI: $0.49-4.3)$ and CPR/cycle $(17 \%, 6 / 36$ and $8 \%, 21 / 275, \mathrm{p}=0.10$; $\mathrm{OR}=2.4 ; 95 \% \mathrm{CI}: 0.91-6.5$ ) were similar in the UTO and control groups. Following OI, 29 (81\%) cycles recruited a lead follicle on the patent fallopian tube, resulting in a CPR/cycle of $21 \%$. In contrast, $19 \%$ of the cycles recruited a lead follicle on the occluded side and, despite hCG trigger and timed intercourse, no pregnancies occurred $(\mathrm{p}=0.3)$. 
Figure 2. Clinical Pregnancy Rates in UTO and Control Groups




Regardless of timed intercourse or treatment, those with a mid-distal or distal occlusion had a higher CPR/patient $(53 \%, 9 / 17)$ compared to those with a proximal occlusion $(29 \%$, $2 / 7$ ), though this finding was not statistically significant $(\mathrm{p}=0.39 ; \mathrm{OR}=$ 2.8; 95\% CI: 0.42-18.8). In those undergoing OI-IUI, the CPR/patient and $\mathrm{CPR} /$ cycle were also higher in those with mid-distal or distal occlusion $(38 \%$, $5 / 13, \mathrm{p}=0.6 ; \mathrm{OR}=3.1 ; 95 \%$ CI: 0.28 35.2 and $23 \% .5 / 22, \mathrm{p}=0.39 ; \mathrm{OR}=3.2$; 95\% CI: 0.33-31.2) compared to those with proximal occlusion $(17 \%, 1 / 6$ and $7 \%, 1 / 12$ ), but also failed to achieve significance.

\section{Section 4: Discussion}

Our data demonstrate that: (1) UTO diagnosed by HSG did not adversely affect CPR, and overall CPR were actually significantly higher in the UTO group than in those undergoing donor insemination with a normal HSG; (2) distal UTO resulted in better pregnancy outcomes than proximal UTO; and (3) OI using oral medication was efficacious with respect to desired recruitment and clinical outcomes.

HSG remains the non-invasive test of choice for determining tubal patency. While HSG is comparable to laparoscopy for determining distal tubal occlusion, laparoscopy is required to definitively diagnose proximal and peritubal disease, as more than a third of patients with abnormal HSG actually have no pathology seen at laparoscopy (Tanahatoe 2008). Traditionally, laparoscopic confirmation and treatment including reconstructive tubal surgery, Copyright @ 2015, Knowledge Enterprises Incorporated. All rights reserved. proximal tubal cannulation, and even salpingectomy of tubal disease has been the standard of care when an abnormal HSG suggesting tubal disease is identified (Tanahatoe 2008). However, laparoscopy is an invasive procedure that is not without known risks (Ebrahimi 2011).

Numerous investigators have advocated for a conservative approach to the management of UTO. Lavy et al. previously reported that for $95 \%$ of patients with a normal HSG or with HSG indicating UTO, laparoscopy did not alter their treatment plan from OIIUI to IVF, suggesting that laparoscopy should be not be immediately recommended in patients with UTO (Lavy 2004). In a retrospective cohort of 359 patients evaluating the prognostic significance of HSG-diagnosed UTO on fertility outcome found that the adjusted pregnancy rates were $0.80 \quad(95 \% \mathrm{CI}$, 0.39-1.6), indicating the limited prognostic significance of UTO compared to bilateral tubal pathology. The median wait time between HSG and laparoscopy was 10 months, while only $5 \%$ of patients with normal or one-sided occluded HSG were then found to have bilateral occlusion at laparoscopy. Since the odds of finding severe bilateral tubal pathology was found to be 1 in 20 even after 10 months, the authors concluded that laparoscopy before 10 months was not justified. Thus, it has been suggested that laparoscopy could be delayed at least 10 months, decreasing the total number of laparoscopies performed as patients even with UTO could become pregnant, and the detection rate of clinically relevant pathology would 
increase (Mol 1999).

Conservative management with OI-IUI has previously been reported in patients with UTO including the use of Gn alone $(n=2)$, a combination of oral therapy and $\mathrm{Gn}(\mathrm{n}=1)$, permutations of $\mathrm{Gn}$ alone, oral alone, and combined $\mathrm{Gn}$ and oral $(\mathrm{n}=1)$, and oral alone $(\mathrm{n}=1)$. For $\mathrm{Gn}$ alone, one study reported similar CPRs for three consecutive cycles in those with suspected UTO $(31 \%, \quad n=55)$ compared to those with unexplained infertility $(42.6 \%, \mathrm{n}=115)$. Pregnancy rates were not significantly different between groups, indicating that conservative treatment may be as appropriate for patients with UTO as it is for infertile patients with normal HSG (Farhi 2007). A second report demonstrated similar CPR of $17.3 \%$ in 133 women with UTO compared to $18.9 \%$ in 570 women with unexplained infertility for one treatment cycle. No cases of PID were reported, and a mean total of $535 \mathrm{IU} /$ patient were used (approximately \$357 USD). No cycle cancellation, OHSS and higher order multiples were discussed (Lin 2013). When using a combination of $\mathrm{CC}$ and human menopausal Gn, Ebrahami et al. also reported similar CPRs per patient for 3 consecutive stimulated IUI cycles in those with suspected UTO $(n=64)$ compared to those with unexplained infertility $(\mathrm{n}=200) \quad(26 \%$ vs $28 \%$, $\mathrm{p}=0.87$ ). No cases of OHSS were reported, but one ectopic occurred (6\%, $1 / 17$ ) in the UTO group. A mean total of $330 \mathrm{IU}$ of $\mathrm{Gn} /$ patient were administered (approximately \$220 $\quad$ USD), with additional monitoring in selected cases.
Cycle cancellation occurred if more than 3 dominant follicles and/or a serum E2 $>1500 \mathrm{pg} / \mathrm{mL}$ were present to avoid OHSS and higher order multiples (Ebrahimi 2011). In the study where a combination of oral, oral and $\mathrm{Gn}$, and $\mathrm{Gn}$ alone were used in conjunction with IUI, Yi et al. reported a similar pregnancy rate per cycle in those with UTO $(17.3 \%, 52$ cycles in 37 patients) compared to those with unexplained infertility $(16.5 \%, 182$ cycles in 114 patients). A mean number of $446 \mathrm{IU}$ of Gn/patient (approximately \$297 USD), were used in $92 \%$ of UTO treated cycles. One ectopic (two in the control group) and one miscarriage (7 in control group) occurred in the study group, and no multiple gestations occurred (Yi 2012). The only study using exclusively CC and UTO compared to unexplained infertility for up to 6 cycles revealed similar PR/cycle $(8.3 \%$ vs $8.2 \%)$ and cumulative PR (38.2\% vs $35.2 \%$ ) after 6 cycles. No mention of CPR, ectopic, or multiple rates were described (Saitou 2010). The financial burden of $\mathrm{Gn}$ ranged from $\$ 200$ to $\$ 357$ in other studies, compared to less than $\$ 100$ in using CC and Letrozole.

Farhi et al. found differences in results based on the location of the tubal occlusion. Pregnancy rates for patients with distal tubal occlusion were 19\%, compared to $38 \%$ in patients with proximal tubal occlusion. The authors concluded that OI-IUI is recommended for patients with unilateral proximal tubal occlusion, but laparoscopy or IVF is recommended for patients with 
unilateral distal tubal occlusion (Farhi 2007). Contrary to results of Farhi's and other studies where pregnancy outcomes are better in those with proximal UTO (range $21 \%$ to $40 \%$ ) compared to distal UTO $(7.7 \%$ to $13.9 \%)$, our results suggest that overall $\mathrm{CP} /$ patient were lower in proximal compared to distal UTO (29\% vs 53\%), though not statistically significantly (Farhi 2007, Lin 2013, Yi 2012). It has been suggested that the detection of proximal tubal occlusion on HSG may have as high as a $15 \%$ false positive rate resulting from tubal spasm, temporary plugs of debris, amorphous material or under-filling of the tube (Lin 2013). With respect to our better outcomes with distal UTO, one could argue that we had fewer false positive reads. HSG interpretations were provided by both the REI physician and radiologist. However, no pregnancies occurred when follicular recruitment occurred on the distal obstructed side.

The limitations of our study include its retrospective nature and the small sample size limiting the ability to generalize our results. However, the strength of our study is our reporting on clinical pregnancies, adding to the body of literature that oral agents are effective for initially managing and treating UTO given the significant economic and health-related disadvantages of laparoscopy and IVF. In contrast to Gn treatment, the lower costs of oral agents and the treatment cycle (ultrasounds and phlebotomy), and the reduction in multiple gestations including higher order multiples, and essentially eliminating the risk of OHSS offer enormous advantages. Cycle cancellation (19\% in our sample) is a factor one should not overlook, but with appropriate counseling and reassurance this can reduce emotional stress when using oral OI-IUI in those with UTO.

In conclusion, a conservative approach in those with UTO appears to provide similar clinical pregnancy success rates compared to patients undergoing donor insemination. Timed intercourse and oral OI-IUI can be considered as a first line approach in the treatment of patients with UTO obviating the need for more aggressive treatments including laparoscopy and IVF. Further studies are needed to clarify the next appropriate intervention including laparoscopy or IVF when using this conservative treatment approach. 


\section{REFERENCES}

Saunders RD, Shwayder JM, Nakajima ST. Current methods of tubal patency assessment. Fertil Steril 2011; 95(7):21719.

Tanahatoe S, Lambalk C, McDonnell J, Dekker J, Mijatovic V, Hompes P. Diagnostic laparoscopy is needed after abnormal hysterosalpingography to prevent over-treatment with IVF. Reprod Biomed Online. 2008 Mar;16(3):410-5.

Lavy Y, Lev-Sagle A, Holtzer H, Revel A, Hurwitz A. Should Laparoscopy be a mandatory component of the infertility evaluation In infertile women with normal hysterosalpingogram or suspected unilateral distal tubal pathology? Eur J Obstet Gynecol Reprod Biol 2004;114(1):64-8.

Farhi J, Ben-Haroush A, Lande Y, Fisch B. Role of treatment with ovarian stimulation and intrauterine insemination in women with unilateral tubal occlusion diagnosed by hysterosalpingography. Fertil Steril 2007: 88(2):396-400.

Lin, Ming-Huei et al. Treatment of infertile women with unilateral tubal occlusion by intrauterine insemination and ovarian stimulation. Taiwan $\mathbf{J}$ Obstet Gynecol 2013: 52(3):360-364.

Yi G, Jee B, Suh C, Kim S. Stimulated intrauterine insemination in women with unilateral tubal occlusion. Clin Exp Reprod Med 2012: 39(2):68-

72.

Berker B, Ye S, Kahraman K, Atabekoglu CS, Sonmezer M, Ozmen B, Ates C. Impact of unilateral tubal blockage diagnosed by hysteroslapingography on the success rate of treatment with controlled ovarian stimulation and intrauterine insemination. J Obsete Gynaecol 2014: 34(2): 127-30.

Saitou Y, Sugimoto K, Saitou M, Hayashi H, Yanaihara N, Tanaka T. Efficacy of Ovarian Stimulation and Intrauterine Insemination in Women with Unilateral Tubal Occlusion Diagnosed by Hysterosalpingography. Jikaikai Med J 2010: 59; 1-4.

Ebrahimi M, Asbagh F, Ghaseminejad A. Controlled ovarian hyperstimulation and intrauterine insemination cycles in patients with unilateral tubal blockage diagnosed by hysterosalpingography. Iran J Reprod Med 2011: 9(1):15-20.

"Side Effects of Injectable Fertility Drugs (gonadotropins)." ASRM.org. American Society for Reproductive Medicine, 2012. Web.

Strandell A, Lindhard A. Why does hydrosalpinx reduce fertility? Hum Reprod 2002: 17(5): 1141-45.

Mol B, Collins J, Burrows E, van der Veen F, Bossuyt, P. Comparison of 
hysterosalpingography and laparoscopy

in predicting fertility outcome. Hum

Reprod 1999: 14 (5): 1237-1242. 